\title{
The Antiviral Activities and Mechanisms of Marine Polysaccharides: An Overview
}

\section{Wei Wang ${ }^{1,2}, *$, Shi-Xin Wang ${ }^{1}$ and Hua-Shi Guan ${ }^{1,2}$}

1 Key Laboratory of Marine Drugs, Ministry of Education, Ocean University of China, Qingdao 266003, China; E-Mails: shixinwang87@ gmail.com (S.-X.W.); hsguan@ouc.edu.cn (H.-S.G.)

2 Shandong Provincial Key Laboratory of Glycoscience \& Glycoengineering, Ocean University of China, Qingdao 266003, China

* Author to whom correspondence should be addressed; E-Mail: wangweidragon @ yahoo.com.cn; Tel.: +86-532-82031980; Fax: +86-532-82033054.

Received: 1 November 2012; in revised form: 26 November 2012 / Accepted: 29 November 2012 / Published: 12 December 2012

\begin{abstract}
Recently, the studies on the antiviral activities of marine natural products, especially marine polysaccharides, are attracting more and more attention all over the world. Marine-derived polysaccharides and their lower molecular weight oligosaccharide derivatives have been shown to possess a variety of antiviral activities. This paper will review the recent progress in research on the antiviral activities and the mechanisms of these polysaccharides obtained from marine organisms. In particular, it will provide an update on the antiviral actions of the sulfated polysaccharides derived from marine algae including carrageenans, alginates, and fucans, relating to their structure features and the structure-activity relationships. In addition, the recent findings on the different mechanisms of antiviral actions of marine polysaccharides and their potential for therapeutic application will also be summarized in detail.
\end{abstract}

Keywords: marine polysaccharides; antiviral activities; mechanisms of action; heparinoid polysaccharide; structure-activity relationship

\section{Introduction}

In recent years, the constant outbreak of some emerging or reemerging viral diseases has caused serious harm to human health. During the last decade, the number of antivirals approved for clinical use 
has been increased from five to more than 30 drugs [1]. Despite these successes, drug efficacy, toxicity, and cost remained unresolved issues, which is particularly large in developing countries due to the relative unavailability of drugs and the continuous emergence of drug resistance. Hence, the development of novel antiviral agents that can be used alone or in combination with existing antivirals is of high importance.

Marine polysaccharides are very important biological macromolecules which widely exist in marine organisms. Marine polysaccharides present an enormous variety of structures and are still under-exploited, thus they should be considered as a novel source of natural compounds for drug discovery [2]. Marine polysaccharides can be divided into different types such as marine animal polysaccharides, plant polysaccharides and microbial polysaccharides according to their different sources. Marine derived polysaccharides have been shown to have a variety of bioactivities such as antitumor, antiviral, anticoagulant, antioxidant, immuno-inflammatory effects and other medicinal properties. In particular, the studies on the antiviral actions of marine polysaccharides and their oligosaccharide derivatives are attracting increasing interests, and marine polysaccharides are paving the way for a new trend in antiviral drugs.

This review presents an overview of recent progress in research on the antiviral activities of marine polysaccharides, relating to their structure features and structure-activity relationships. Moreover, this review will mainly focus on the heparinoid polysaccharides and the sulfated polysaccharides present in seaweed. Recent developments in the mechanisms of antiviral actions of marine polysaccharides and their oligosaccharide derivatives will also be discussed in detail.

\section{The Classification and Main Structure Features of Marine Polysaccharides}

Marine polysaccharides usually exhibit structural features such as sulfate and uronic acid groups, which distinguish them from polysaccharides of terrestrial plants, but are similar to mammalian glycosaminoglycans, such as heparin and chondroitin sulfate [3]. Marine polysaccharides can be classified into three main types: marine animal polysaccharides, marine plant polysaccharides, and marine microbial polysaccharides according to their different sources, and each have different structure features.

\subsection{The Main Structure Features of Marine Animal Polysaccharides}

Marine animals are rich in polysaccharides, and the polysaccharides derived from marine fishes, shellfishes, and mollusks often possess a wide range of pharmacological activities [4]. The marine animal polysaccharides usually include chitosans derived from crustaceans, chondroitin sulfates from cartilaginous fishes, sulfated polysaccharides from sponge, and glycosaminoglycans from scallops and abalone [5,6].

Chitin, a long-chain polymer of $\mathrm{N}$-acetylglucosamine, is one of the most abundant polysaccharides and usually prepared from the shells of crabs and shrimps [7,8]. Chitosan, a partially deacetylated polymer of $\mathrm{N}$-acetylglucosamine, is produced commercially by deacetylation of chitin [9]. The molecular weight of commercially produced chitosan is usually between 3800 and 20,000 Daltons. Chitosan is a linear randomly distributed, hetero polysaccharide consisting of $\beta$-( $1 \rightarrow 4)$-linked 2-acetamido-2-deoxy-D-glucopyranose and 2-amino-2-deoxy-D-glucopyranose units [2]. Chemical 
modification of chitin and chitosan can generate new biofunctional products which possess good biological activities and physicochemical properties [10-14]. Moreover, it was reported that the marine polysaccharides isolated from cartilaginous fishes also contain trace neutral mannose, xylose and rhamnose besides the galactosamine and glucuronic acid, which have certain structure-specific properties [15].

Furthermore, Cimino et al. [16] reported that rosacelose, a new anti-HIV polysaccharide composed of glucose and fucose sulfate, could be isolated from the aqueous extract of the marine sponge Mixylla rosacea. They also found that this marine polysaccharide has a linear polysaccharide structure mainly composed of 4,6-disulfated 3-O-glycosylated $\alpha$-D-glucopyranosyl and 2,4-disulfated 3-O-glycosylated $\alpha$-L-fucopyranosyl residues (in a 3:1 molar ratio) [16]. Moreover, it was reported that one kind of sugar polymer which contains hexosamine, hexuronic acid, and fucose sulfate could be separated from Apostichopus japonicus selenka [17]. In a word, marine animal polysaccharides have extremely broad distribution, and exist in almost all marine animal tissues and organs.

\subsection{The Main Structure Features of Marine Plant Polysaccharides}

The marine plant polysaccharides especially the seaweed polysaccharides are widely distributed in the ocean, occurring from the tide level to considerable depths, free-floating or anchored, which are the most abundant polysaccharides in marine organisms. Moreover, the polysaccharide content of seaweed is very high, accounting for more than $50 \%$ of the dry weight, thus the seaweed polysaccharides are very important resources for the development of marine polysaccharide drugs. The principal cell wall polysaccharides in green seaweeds are ulvans, those in red seaweeds are agarans and carrageenans, and those in brown seaweeds are alginates and fucans, as well as the storage polysaccharide laminarin $[18,19]$.

Alginates are the major constituent of brown seaweeds' cell walls and are linear acidic polysaccharides composed with a central backbone of poly-D-glucuronic acid (G blocks), poly-D-mannuronic acid (M blocks) and alternate residues of D-guluronic acid and D-mannuronic acid (GM blocks) [20]. Fucans are also one of the major constituents of brown seaweed cell walls, and are ramified sulfated polysaccharides constituted by a central backbone of fucose sulfated in positions C2 and/or C4 and ramifications at each two or three fucose residues [21].

Red seaweed polysaccharides are primarily classified as agarans and carrageenans based on their stereochemistry, specifically galactans with 4-linked $\alpha$-galactose residues of the L-series are termed agarans, and those of the D-series are termed carrageenans [22]. Carrageenans are sulfated D-galactans composed of repeating disaccharide units with alternating 3-linked $\beta$-D-galactopyranose (G-units) and 4-linked $\alpha$-galactopyranose (D-units) or 3,6-anhydro- $\alpha$-galactopyranose (AnGal-units), which possess broad-spectrum antiviral activities [18]. In conclusion, the seaweed polysaccharides are the most abundant polysaccharides in marine plants, and usually possess the special characteristics of high sulfation and carboxylation.

\subsection{The Main Structure Features of Marine Microbial Polysaccharides}

Marine microorganisms, including bacteria, fungi, and microalgae, are of considerable importance as promising new sources of a huge number of biologically active products [23-26]. Some of these marine 
species live in high-pressure, high-salt, low-temperature, and oligotrophic environments, which provide the opportunity for them to produce unique active substances that differ from the terrestrial ones [27].

Marine microbial polysaccharides, especially the extracellular polysaccharides, have structural diversity, complexity, and particularity. Most of these polysaccharides are heteropolysaccharides which composed by different monosaccharides in a certain percentage, wherein the glucose, galactose and mannose are the most common components in microbial polysaccharides. It was reported that spirulan, a sulfated polysaccharide isolated from Arthrospira platensis (formely Spirulina platensis) is composed of two types of disaccharide repeating units, $[\rightarrow 3)-\alpha$-L-Rha $(1 \rightarrow 2)-\alpha$-L-Aco- $(1 \rightarrow]$ where Aco (acofriose) is 3-O-methyl-Rha with sulfate groups and $O$-hexuronosyl-rhamnose [28].

In addition, the marine microbial polysaccharides also contain glucuronic acid, galacturonic acid, amino sugars, and pyruvate. Roger et al. [29] reported that the exopolysaccharide (EPS) derived from marine bacterium Alteromonas infernus is a highly branched acidic heteropolysaccharide with a high molecular weight and low sulfate content (less than 10\%). Its nonasaccharide repeating unit is composed of uronic acid (galacturonic and glucuronic acid) and neutral sugars (galactose and glucose), and substituted with one sulfate group [29]. In conclusion, the marine microbial polysaccharides with novel chemical compositions and structure features have been found to possess potential applications in fields such as pharmaceuticals, food additives, and industrial waste treatments.

\section{The Antiviral Effects and Structure-Activity Relationships of Marine Polysaccharides}

\subsection{The Antiviral Activities of Carrageenan Polysaccharides and Their Oligosaccharide Derivatives}

Carrageenans are one of the major constituents of red seaweed cell walls representing $30 \%$ to $75 \%$ of the algal dry weight [30]. Carrageenan is mainly extracted from certain genera of red seaweeds such as Chondrus, Gigartina, Hypnea, and Eucheuma, and is currently the most widely studied red algae polysaccharide [31]. Carrageenan polysaccharides are normally classified according to their structural characteristics, including their sulfation patterns and the presence or absence of 3,6-anhydro bridges in $\alpha$-linked galactose residues [18].

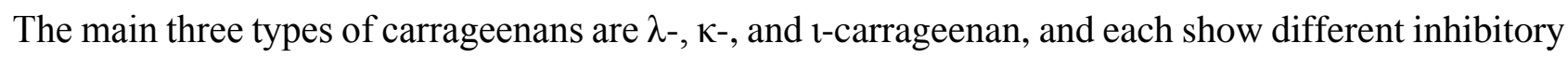
effects on different viruses [32-37]. Buck et al. demonstrated that carrageenans could inhibit the initial infection process of human papillomavirus (HPV), and the antiviral effect of $t$-carrageenan is better than that of $\lambda$ - and $\kappa$-carrageenans [38]. Talarico and co-workers reported that $\mathrm{t}$-carrageenan could inhibit dengue virus (DENV) replication in mammalian and mosquito cells, and the mode of action of t-carrageenan in both cell types is strikingly different. [33,37]. Yamada and co-workers reported that $O$-acylated carrageenan polysaccharides with different molecular weights had increased anti-HIV activity by depolymerization and sulfation [39,40]. Thus, the antiviral activities of carrageenan polysaccharides are associated with their molecular weights and the content of sulfates. Despite having good inhibitory effects on virus infection, the high molecular weight (MW) and poor tissue-penetrating ability of carrageenan polysaccharides have limited potential antiviral application in humans.

Carrageenan oligosaccharide prepared from carrageenan by chemical or enzymatic degradation has smaller molecular weight and is easy to contact with viruses, thus its bioavailability and biological activity is significantly increased [41]. Tang et al. found that low molecular weight carrageenans and 
their derivatives showed significant inhibition effects against influenza virus FM1-induced pulmonary edema in mice, and the $3 \mathrm{kDa} \mathrm{k}$-carrageenan with proper acetylation degree and sulfation degree possesses best antiviral activity in vivo [42]. Wang and co-workers reported that the low molecular weight carrageenan oligosaccharide $\mathrm{KCO}$ and their sulfated derivatives could effectively inhibit influenza A virus (IAV) replication in vitro and in vivo, and KCO mainly inhibits a replication step in IAV life cycle subsequent to viral internalization but prior to virus release $[43,44]$. In addition, the structure-activity relationship study of KCO showed that the specific sugar linkage, a certain sugar length and sulfate content might be essential for the anti-IAV activity of k-carrageenan oligosaccharides. The most active k-carrageenan oligosaccharide has a sulfate content of $0.8-1.0 \mathrm{~mole} / \mathrm{mole}$ of disaccharide and a molecular weight of $1-3 \mathrm{kDa}$ [44].

In conclusion, the antiviral activities of carrageenans are very broad, which can suppress the replication of both enveloped and non-enveloped viruses. The antiviral effects of carrageenans are closely related to the molecular weights and the degree of sulfation of them. Moreover, the inhibitory actions of carrageenans on different viruses are usually different, which are associated with the types of carrageenans, the virus serotypes and the host cell itself [45-48].

\subsection{The Antiviral Activities of Heparin and Heparinoid Polysaccharides}

Heparin is a member of the glycosaminoglycan (GAG) family of carbohydrates and consists of a variably sulfated repeating disaccharide unit. The most common disaccharide unit is composed of 2-O-sulfate-alpha-L iduronic acid and 6- $O$-sulfate- $N$-sulfate- $\alpha$-D glucosamine linked by $1 \rightarrow 4$ glycosidic bond. Heparin has been used in clinical practice for nearly 70 years, and is mainly used for anticoagulant and the treatment of thrombotic diseases. In addition to the anticoagulant and antithrombotic activities, heparin also possesses the inhibitory effect on smooth muscle cell proliferation, anti-inflammatory effect, antitumor effect, and the antiviral activities [49-52]. Some studies indicated that the heparin polysaccharides and the low molecular weight heparin could be used as the natural inhibitors of herpes simplex virus type 1 (HSV-1), and the inhibition of HSV-1 infection by heparin requires a unique sulfation moiety [52,53].

The marine heparinoid polysaccharides are similar to heparin in structure, and possess GAG-like biological properties, which contain alginates, ulvans, and their sulfated derivatives, as well as the dextran sulfate and chitosan sulfate (Figure 1). Recent studies showed that the cell surface heparinoid sulfate proteoglycans are the initial receptors of human herpes virus HSV-1, HSV-2, and bovine herpes virus in their infection processes [54-56]. Heparinoid polysaccharides can interact with the positive charge regions of cell surface glycoproteins, leading to the shielding effect on these regions, thus preventing the binding of viruses to the cell surface [54].

Madeleine et al. reported that the dextran sulfate could inhibit the fusion of influenza virus with cell membranes, and suppress the replication of influenza virus in vivo [57]. Witvrouw et al. [58] found that the dextran sulfate mainly inhibits the replication of enveloped viruses, but is inactive against non-enveloped viruses. In addition, the low molecular weight dextran sulfate (DS1000) is virtually inactive against HSV, influenza virus, and some other viruses, and the inhibitory effect of DS1000 against HIV varies considerably depending on the virus strain and the cell type [58]. Moreover, Ivanova et al. noted that the ulvan polysaccharides isolated from green algae had good inhibitory effect 
on influenza A virus, and the inhibition effect is dose-dependent and strain-specific [59]. In addition, another heparinoid polysaccharide — chitosan sulfate also possesses good antiviral activities [60].

In summary, the marine heparinoid polysaccharides have similar pharmacological activities to the natural heparin, and all can inhibit the infection and replication of different kinds of viruses.

Figure 1. Idealized average structures of the repeat units of heparin and heparinoid polysaccharides $[18,38,58,60]$.

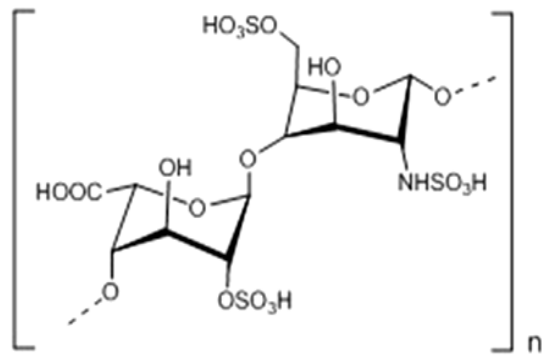

Heparin

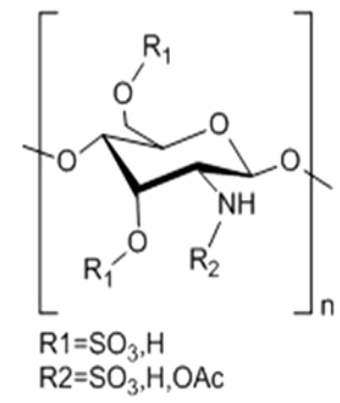

Chitosan Sulfate

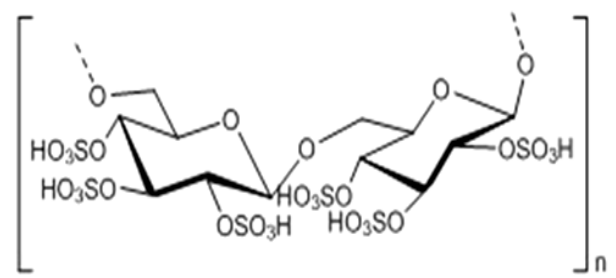

Dextran sulfate

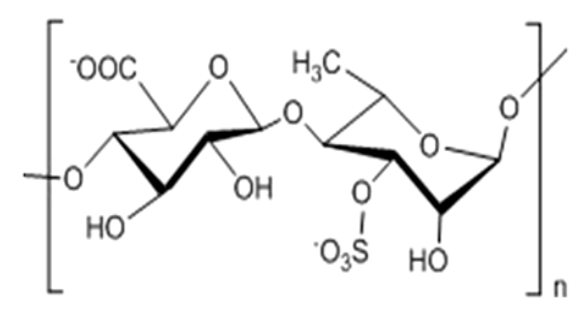

Ulvan (Ulvanobiouronic acid)

\subsection{The Antiviral Activities of Alginate Polysaccharides and Their Sulfated Derivatives}

The principal cell wall polysaccharides in brown seaweeds are alginates and fucans as well as the storage polysaccharide laminarin. Alginate polysaccharides are mainly extracted from brown seaweeds such as kelp, macroalgae, bubble zosteraceae, and marjoram algae, and have been widely used in the food, cosmetic and pharmaceutical industries as a thickener and gelling agent. Alginates are the major constituent of brown seaweeds, which composed of a central backbone of poly-D-glucuronic acid, poly-D-mannuronic acid and alternate residues of D-guluronic acid and D-mannuronic acid [20].

Xin et al. reported that a marine polysaccharide drug 911 derived from alginate could significantly inhibit the acute infection of MT4 cells and the chronic infection of H9 cells with HIV-1 [61]. 911 can significantly inhibit the replication of HIV in vitro and in vivo, and its inhibitory action is attributed to the inhibition of viral reverse transcriptase, the interference with viral adsorption, and the enhancement of immune function $[61,62]$. Geng et al. found that the sulfated polymannuroguluronate (SPMG) could inhibit HIV adsorption mainly through interfering with the interaction of virus gp120 protein with the CD4 molecule on the surface of $T$ cells [63-65]. In addition, their studies also indicated that the octasaccharide unit is established to be the minimal active fragment of SPMG inhibiting syncytium 
formation and lowering the P24 antigen level in HIV-IIIB-infected CEM cells [65]. Moreover, 911 can improve the immune function of host cells, and inhibit the activity of hepatitis B virus (HBV) DNA polymerase, thus 911 can also be used to inhibit the replication of HBV [66].

Fucans are a class of high molecular weight sulfated polysaccharides, which can be classified into three major groups: fucoidans, xylofucoglycuronans and glycuronogalactofucans. They are widely distributed in several species of brown algae and composed of a mainly repeating chain of fucose [60]. The sulfated fucans from the seaweed species Dictyota mertensii, Lobophora variegata, Spatoglossum schroederi and Fucus vesiculosus were reported to be able to inhibit the activity of HIV reverse transcriptase (RT) [67]. A fucan polysaccharide from Cladosiphon okamuranus composed of glucuronic acid and sulfated fucose units can inhibit the infection of BHK-21 cells with dengue virus type 2 (DENV-2), but shows little effect on the other three serotypes of the virus (DENV-1, DENV-3 and DENV-4) [68]. Sulfation of the fucan is necessary for this activity, and carboxyl-reduction of the glucuronic acids to glucose units abolishes the fucans' antiviral activities. Moreover, Akamatsu et al. reported that one new type of fucose polysaccharides called "MC26," which is extracted from brown seaweed, has excellent anti-influenza A virus effect in vitro and in vivo [69].

Laminaran (or laminarin) was first discovered in the laminaria species and appears to be the food reserve of all brown algae. Laminaran is a water-soluble polysaccharide containing 20-25 glucose units composed of $(1,3)-\beta$-D-glucan with $\beta(1,6)$ branching. Laminaran isolated from some brown algae has a good inhibitory effect on virus proliferation. The laminaran polysaccharides prepared from kelp by water extraction were reported to be able to effectively inhibit the adsorption of HIV on lymphocytes and the activity of HIV reverse transcriptase at the concentration of $50 \mu \mathrm{g} / \mathrm{mL}$, which suggest that laminaran polysaccharides possess good inhibitory effect on HIV replication [70].

In conclusion, brown algae-derived polysaccharides and their derivatives, in particular the alginate polysaccharides and fucans, have good antiviral activities, thus the alginate polysaccharides have the potential to be developed into a new type of antiviral drugs with high efficiency and low toxicity.

\subsection{The Antiviral Activities of Marine Shellfish Polysaccharides and Their Derivatives}

The polysaccharides isolated from marine animals usually have the characteristics of high sulfation, and are also the important resources for the development of antiviral drugs. The marine shellfish polysaccharides are the important animal polysaccharides isolated from marine shellfishes, which commonly including abalone polysaccharides, mussels polysaccharides, and clams polysaccharides. The structure of marine shellfish polysaccharides is very complex, not only because of the wide variety of monosaccharide compositions, but also because of the diversity of linkage types (with or without branches).

Yu et al. [71] studied the inhibition actions of scallop skirt glycosaminoglycan (SS-GAG) on the type I-herpes simplex virus (HSV-I) at different concentrations, and they found that SS-GAG has a significant anti-HSV-1 effect in vitro. The antiviral activity of SS-GAG gradually increases with the prolonged duration of action [71]. Zhang et al. found that marine polysaccharides isolated from Perna viridis could significantly inhibit the replication of influenza virus in MDCK cells, and has an additive effect on the anti-IAV actions of ribavirin, which suggest that Perna viridis polysaccharides 
merit further investigation as novel anti-influenza virus agents that can be used alone or in combination with existing antivirals [72].

Moreover, Wu et al. reported that the oyster polysaccharides could inhibit the DNA replication of duck hepatitis B virus (DHBV), and reduce the content of DHBV-DNA in duck serum, thus having obvious anti-HBV effects in vivo [73]. In addition, Woo et al. investigated the inhibition effects of marine shellfish polysaccharides derived from seven kinds of shellfish (Ruditapes philippinarum, Mytilus coruscus, Scapharca broughtonii, Scapharca subcrenata, meretrix lusoria, Meretrix petechialis and Sinonovacula constricta Lamark) on the infection of HIV-1 in vitro, and the results showed that the seven species of marine shellfish polysaccharides all could inhibit the fusion of virus gp120/gp41 with CD4 protein on the surface of $\mathrm{T}$ lymphocytes, of which the Meretrix petechialis polysaccharide possesses the most significant anti-HIV activity [74].

In conclusion, a variety of marine active polysaccharides with novel structures and unique functions have been obtained from marine shellfishes, and these polysaccharides are expected to become the new resources for the antiviral drugs development.

\subsection{The Antiviral Activities of Chitosan Polysaccharides and Their Derivatives}

Chitosan, a partially deacetylated polymer of $\mathrm{N}$-acetylglucosamine, is produced commercially by deacetylation of chitin [9]. Chitosans were found to effectively reduce the infectivity of feline calicivirus FCV-F9 and bacteriophages MS2 and phiX174. Reduction of MS2 infectivity by chitosan increases as the molecular weight of chitosan increases, with high molecular weight chitosan (over $200 \mathrm{kDa}$ ) being able to completely reduce titers of MS2 [75]. Moreover, chitosan was reported to be able to inhibit the systemic propagation of viruses and viroids throughout the plant and enhance the host's hypersensitive response to infection [76-79]. In addition, the level of suppression of plant virus infections varies according to the species of plants and the chitosan sources [78]. Furthermore, it was reported that the antiviral activities of the low molecular weight chitosans produced by enzymatic degradation significantly increase with the lowering of their polymerization degree $[77,79]$.

Chemical modification of chitin and chitosan can generate novel compounds that possess good pharmacological properties such as antiviral activities. Sosa et al. reported that the $\mathrm{N}$-carboxymethylchitosan $\mathrm{N}, \mathrm{O}$-sulfate (NCMCS), a polysaccharide derived from $\mathrm{N}$-carboxymethyl chitosan by sulfation modification, could prevent HIV-1 infection by inhibiting viral adsorption to the CD4 receptor and reverse transcription of the viral genome [80]. Moreover, Nishimura et al. found that the chitin sulfate had good inhibitory effect on HIV-1 infection, and its inhibition action on HIV-1 depends significantly on the sites of sulfation [81]. In addition, it was reported that the aminoethyl-chitosan, prepared from $50 \%$ deacetylated chitosan also shows good inhibitory activity against HIV-1 in vitro [60].

To improve the water-solubility and biological activity, chitosan also can be converted into chitooligosaccharides (COSs) via either chemical or enzymatic hydrolysis [82-84]. Sulfated chitooligosaccharides (SCOS), prepared by a random sulfation modification of chitooligosaccharides, have been reported to possess good anti-HIV activities at low molecular weight (3-5 kDa) [85]. SCOS shows inhibitory actions on viral entry and virus-cell fusion via blocking the interaction between 
HIV-1 gp120 and $\mathrm{CD}^{+}$cell surface receptors. Moreover, the administration of chitooligosaccharides on different leaf surfaces can decrease the number of local necroses, which is virus-induced infection [86].

In summary, chitosan polysaccharides and low molecular weight oligosaccharides, as well as their sulfated derivatives, possess good antiviral activities, and their antiviral actions are related to the molecular weights and their sites of sulfation.

\subsection{The Antiviral Activities of Polymannuronic Acid Polysaccharides and Their Derivatives}

Alginates are linear acidic polysaccharides constituted by a central backbone of poly-D-glucuronic acid (G blocks), poly-D-mannuronic acid (M blocks) and alternate residues of D-guluronic acid and D-mannuronic acid (GM blocks) [20]. The alginate polysaccharide dominated by heterogeneous fragments (MG) has been developed into the novel anti-HIV marine drug 911, which has entered Phase II clinical study [62,64]. The alginate polysaccharide composed of homogeneous fragments (M blocks) has been developed into the anti-cardiovascular marine drug polymannuronic acid propyl sulfate (PMS) [87]. Polymannuronic acid polysaccharides are alginic acid sugar fragments (M blocks) prepared from alginate polysaccharides by enzymatic or acidic degradation and $\mathrm{PH}$ fractionation method. The chemical structures of alginate polysaccharides and their derivatives are illustrated in Figure 2.

Figure 2. Chemical structures of alginate polysaccharides and their derivatives [20,64,87].

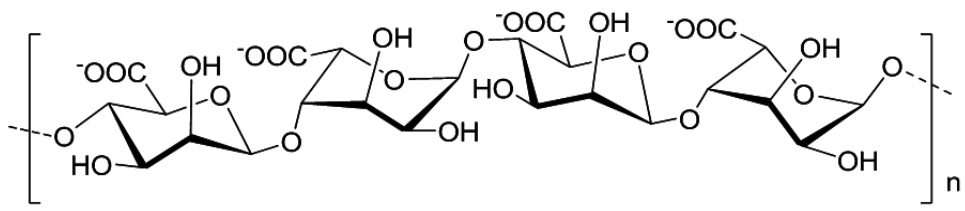

Alginate polysaccharide (GM blocks)

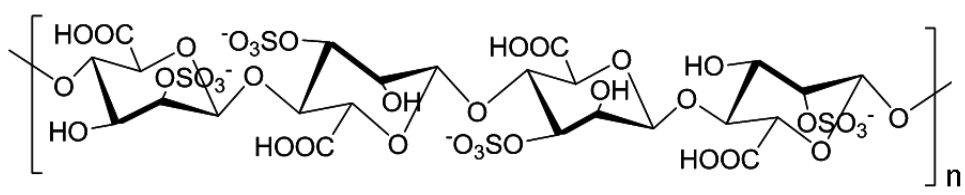

Sulfated polymannuroguluronate (SPMG)

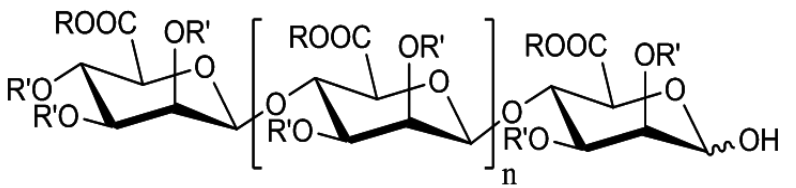

$\left(\mathrm{R}=\mathrm{Na}, \mathrm{CH}_{2} \mathrm{CHOHCH}_{3} ; \mathrm{R}^{\prime}=\mathrm{SO}_{3} \mathrm{Na}, \mathrm{H}\right)$

Polymannuronic acid propyl sulfate (PMS)

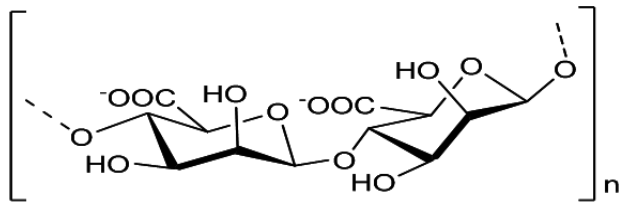

Oligomeric mannuronic acid (OM)

Polymannuronic acid polysaccharides and their derivatives have heparin-like structures, and also possess good antiviral activities. It was reported that one derivative of polymannuronic acid 
polysaccharides-polymeric mannuronic acids sulfate that was prepared by the sulfation of the polymannuronic acid fragment-possesses good antiviral effects in vivo [88]. Guan and co-workers found that polymeric mannuronic acids sulfate could significantly improve the humoral and cellular immune function, and effectively reduce the surface antigen levels and the DNA levels of hepatitis B virus in duck blood, suggesting that this polysaccharide has good anti-duck HBV effect [88].

Furthermore, it was reported that one oligosaccharide derivative of polymannuronic acid polysaccharide - oligomeric mannuronic acid (OM) with low molecular weight (less than $5 \mathrm{kDa}$ ) has good inhibitory effects on the infection of influenza A (H1N1) virus in vitro and in vivo [89]. Guan and co-workers reported that another derivative of polymannuronic acid-polymannuronic acid propyl sulfate (PMS) could be prepared by the sulfation and propyl modification of polymannuronic acid polysaccharide, which has the heparin-like structural characteristics [87]. Moreover, they also found that PMS has good inhibition effect on the neuraminidase activity of influenza A virus (IAV), and effectively inhibits IAV infection in MDCK cells. Additionally, PMS can effectively alleviate the pneumonia symptom caused by IAV infection, and significantly reduce the mortality rate of mice [90].

In conclusion, polymannuronic acid polysaccharides and their oligosaccharide derivatives possess good antiviral activities, which merit further investigation as novel antiviral agents to prevent the infection of HBV and IAV. In order to summarize the data available in the literature, the marine polysaccharides with good antiviral effects described in this paper were all shown in Table 1.

Table 1. Antiviral activities of selected marine polysaccharides.

\begin{tabular}{cccc}
\hline Marine organisms & Specific polysaccharides & Antiviral effects & References \\
\hline \multirow{2}{*}{ Crustacean } & Chitosan & $\begin{array}{c}\text { Anti-enteric virus, } \\
\text { plant viruses, HIV }\end{array}$ & {$[75-81,85,86]$} \\
\hline \multirow{2}{*}{ Red algae } & $\lambda$-carrageenan & $\begin{array}{c}\text { Anti-DENV, HSV, HPV, } \\
\text { HIV, HAV }\end{array}$ & {$[33,35,38-40,46,47]$} \\
\cline { 2 - 4 } & א-carrageenan & $\begin{array}{c}\text { Anti-enterovirus, } \\
\text { HSV, HIV, IAV }\end{array}$ & {$[32,35,39,40,42-44]$} \\
\cline { 2 - 4 } & 1-carrageenan & $\begin{array}{c}\text { Anti-DENV, HRV, IAV, }, \\
\text { HPV, HAV }\end{array}$ & {$[33,34,36-38,47,48]$} \\
\hline \multirow{2}{*}{ Brown algae } & Alginate & Anti-HIV, HBV, IAV & {$[61-66,88-90]$} \\
\cline { 2 - 4 } & Fucan & Anti-HIV, DENV, IAV & {$[67-69]$} \\
\cline { 2 - 4 } Green algae & Laminarin & Anti-HIV & {$[70]$} \\
\hline \multirow{2}{*}{ Shellfish } & Ulvan & Anti-IAV & {$[59]$} \\
\hline Microalgae & Shellfish polysaccharide & $\begin{array}{c}\text { Anti-HSV, IAV, } \\
\text { HBV, HIV }\end{array}$ & {$[71-74]$} \\
\hline
\end{tabular}

\section{The Mechanisms of Antiviral Actions of Marine Polysaccharides}

Marine polysaccharides can either inhibit the replication of virus through interfering viral life cycle or improve the host antiviral immune responses to accelerate the process of viral clearance. The life cycle of viruses differs greatly between species but there are six basic stages in the life cycle of viruses: viral adsorption, viral penetration, uncoating of capsids, biosynthesis, viral assembly and viral release. 
Marine polysaccharides can inhibit viral life cycle at different stages or directly inactivate virions before virus infection. Specific antiviral mechanism of marine polysaccharides is commonly related to specific structure features of the polysaccharides and specific viral serotypes [45].

\subsection{Direct Virucidal Action}

Carrageenan, a sulfated polysaccharide, might inhibit virus infection via direct actions on the virus surface by its negative charge. Several studies showed that carrageenan has a direct virucidal action on some enveloped viruses, which makes the viruses lose the ability to infect cells, thus effectively reducing the virus multiplication. Carlucci et al. found that $\lambda$-type carrageenan could firmly bind to the herpes simplex virus (HSV), leading to the inactivation of theHSV virion, thus inhibiting the replication of HSV [93]. Their studies further suggest that carrageenan changes the structure of the glycoproteins $\mathrm{gB}$ and $\mathrm{gC}$ of HSV [46,93].

Moreover, Harden et al. [94] reported that carrageenan polysaccharides derived from red algae could directly inactivate HSV-2 at low concentrations. The virucidal activities increase with increased molecular weight of carrageenan polysaccharide up to $100 \mathrm{kDa}$, after which the virucidal activities level off [94]. The direct virucidal actions of carrageenan may be due to the formation of a stable virion-carrageenan complex where binding is not reversible and hence the sites on the viral envelope required for virus attachment to host cells are occupied by the sulfated polysaccharide, which renders the virus unable to complete the subsequent infection process [45].

In addition, chitosan and chitosan oligosaccharides were reported to be able to effectively reduce the infectivity of two human enteric viral surrogates: feline calicivirus F-9 (FCV-F9), and bacteriophage MS2 (MS2) by incubating the two viruses with chitosan or chitosan oligosaccharides for $3 \mathrm{~h}$ at $37{ }^{\circ} \mathrm{C}$ before infection [75,95]. The inhibition actions might be due to the direct inactivation of these two enteric viral surrogates by chitosan. Furthermore, reduction of MS2 infectivity by chitosan increases as the molecular weight of chitosan increases, while the inhibitory effects of chitosan on FCV-F9 is not MW-dependent [95].

In conclusion, chitosan and carrageenan polysaccharides all have direct virucidal actions on some viruses to block the virus infection. This virucidal activity is important for marine polysaccharides since it is associated with the augmentation of antiviral activities in vivo [35].

\subsection{Inhibition of Viral Adsorption}

The first step of virus invasion into host cells is binding to host cell surface via electrostatic interactions, and then transforming unstable reversible binding into stable irreversible adsorption in order to achieve subsequent invasion process. Some marine polysaccharides can interfere with viral adsorption process either by interacting with virions or by interacting with virus receptors on the host cell surface.

Several studies have shown that carrageenan can mask the positive charge of host cell surfaces by the negative charge of its sulfate groups, so as to interfere with the adsorption process of viruses. Mazumder et al. obtained a high molecular weight sulfated galactan from red algae, and showed its antiviral activities against herpes simplex virus 1 and 2 in bioassays, which is likely due to an inhibition of the initial viral attachment to the host cells [96]. Carlucci et al. noted that $\lambda$-carrageenan and partially 
cyclized $\mu / \mathrm{l}$-carrageenan from Gigartina skottsbergii have potent antiviral effects against different strains of HSV types 1 and 2 during the virus adsorption stage [97]. They subsequently confirmed the firm binding of carrageenan to virus receptors on the host cell surface. Their studies demonstrate that $\lambda$-carrageenan interferes with the adsorption process of the virus to the host cell surfaces [97,98].

Fucoidan isolated from brown algae has good antiviral activities against viruses such as HIV [99], HSV and human cytomegalovirus [100-103]. The antiviral actions of fucoidan seem to stem from inhibiting the binding of the virus particles to the host cell [103]. In addition, sulfated polymannuroguluronate (SPMG), a new form of marine polysaccharide extracted from brown algae, could inhibit HIV-1 adsorption and entry by sharing common binding sites on gp120 with sCD4 or masking the docking sites of gp120 for sCD4 on the surface of T lymphocytes [64].

Furthermore, one of the chitosan derivatives, $N$-carboxymethylchitosan $N, O$-sulfate (NCMCS), could inhibit the replication of HIV-1 by blocking the interactions of viral coat glycoprotein receptors with target proteins on lymphocytes [80]. In addition, Kanekiyo et al. [91] reported that the acidic polysaccharide nostoflan isolated from the edible blue-green alga Nostoc flagelliforme have a good inhibitory effect on HSV-1. Their results indicate that the inhibition of virus binding to - but not penetration into-host cells is responsible for the antiherpetic effect induced by nostoflan [91]. In summary, marine polysaccharides from different sources can block virus infection by interfering with the virus adsorption process.

\subsection{Inhibition of Virus Internalization and Uncoating}

The internalization process of most animal viruses often involves the endocytic uptake, vesicular transport through the cytoplasm, and delivery to endosomes and other intracellular organelles [104]. The uncoating step of the viral life cycle usually occurs after viral internalization into host cells, while the uncoating of some phages occurs in the same time of internalization. Certain marine polysaccharides, especially sulfated marine polysaccharides, can interfere with virus internalization and uncoating by blocking the allosteric process of virus particles.

Buck et al. found that carrageenan could directly bind to the HPV capsid, so as to inhibit not only the viral adsorption process but also the subsequent entry and uncoating process of the virus [38]. They also found that the inhibition actions of carrageenan against HPV might be related to a mechanism that is independent of the heparan sulfate after viral adsorption [38]. Moreover, Talarico and co-workers reported that $\lambda$ - and $\mathrm{t}$-carrageenans could interfere with both DENV-2 adsorption and internalization into host cells and are only effective if added together with the virus or shortly after infection [33]. The mechanism of this inhibition action may be due to that although DENV virus can enter into host cell in the presence of carrageenans, their subsequent uncoating and releasing from endosomes may be interfered by the carrageenans. The inhibition action of l-carrageenan on the uncoating process of dengue virus may be attributed to the direct interaction of carrageenans with the virus membrane glycoprotein E (gE) [33,48,105].

Moreover, Grassauer et al. reported that $\mathrm{l}$-carrageenan could inhibit rhinovirus (HRV) multiplication by interfering with the very early stages of virus replication, and this inhibition is probably due to the interference of the allosteric process of virus particles during HRV internalization [34]. Furthermore, Kim et al. reported that the sulfated polysaccharide, p-KG03, purified from the marine microalga, 
Gyrodinium impudium, exhibit good inhibitory effect on influenza A virus infection [92]. The mechanism studies showed that the inhibition of virus replication is maximized when p-KG03 is added during or within $6 \mathrm{~h}$ after viral infection, suggesting that mainly the viral adsorption and internalization steps are targeted by this compound. Thus the marine polysaccharide p-KG03 cannot only inhibit the binding of influenza virus to host cells, but also prevents the cellular internalization of the virus and early viral replication [92].

In conclusion, some marine polysaccharides can interact with virus membrane glycoproteins to interfere with virus internalization and the subsequent uncoating and releasing from endosomes in the cytoplasm in order to inhibit virus multiplication in vitro.

\subsection{Inhibition of Virus Transcription and Replication}

Marine polysaccharides, especially the low molecular weight marine oligosaccharides, cannot only block the virus invasion process, but also inhibit the virus transcription and replication processes after internalization into host cells. The inhibition of virus transcription and replication may be related to the direct interference of viral replication enzymes or on other intracellular targets by marine polysaccharides.

Gonzalez et al. used $\left[{ }^{35} \mathrm{~S}\right]$ methionine-labeled virions to analyze the internalization of HSV-1 into host cells, and found that carrageenan polysaccharides have no inhibitory effect on virus adsorption or virus entry, but the subsequent protein synthesis of virus is declined [106]. However, the expression of viral late proteins could not be affected by the carrageean polysaccharides if the compounds are added to host cells one hour after virus infection, which suggest that this sulfated polysaccharide inhibits a step in virus replication subsequent to viral internalization but prior to the onset of late viral protein synthesis [106]. In addition, Talarico et al. found that 1 -carrageenan could inhibit the replication of DENV in mosquito cells. Its mechanism of action is not to suppress the virus adsorption process but to affect the potential targets within the host cells [37].

Moreover, carrageenan oligosaccharides with low molecular weights can also inhibit virus transcription and replication process after entering into host cells. Wang et al. found that $\kappa$-carrageenan oligosaccharide with low molecular weight could effectively inhibit influenza A H1N1 virus replication in vitro and in vivo $[43,44]$. They also noted that carrageenan oligosaccharide $\mathrm{CO}-1$ does not bind to the cell surface of MDCK cells and does not interfere with the IAV adsorption and internalization process directly [43]. Different to the actions of carrageenan polysaccharides, the oligosaccharide CO-1 could enter into host cells and inhibit IAV mRNA transcription and protein translation after its internalization. In a word, carrageenan oligosaccharide CO-1 might inhibit an early replication step of virus life cycle subsequent to IAV internalization [43].

In addition, some marine polysaccharides derived from brown algae can also inhibit virus replication in host cells. Queiroz et al. found that fucans isolated from $F$. vesiculosus have a pronounced inhibitory effect in vitro on the HIV reverse transcriptase at a concentration of $0.5-1.0 \mathrm{mg} / \mathrm{mL}$, and the inhibitory effect of fucans is eliminated by their desulfation [67]. Queiroz and co-workers also indicated that the antiviral activity of fucans is not only dependent on the ionic changes but also on the sugar rings that act to spatially orientate the charges in a configuration that recognizes the enzyme, thus determining the specificity of the binding [67]. In addition, the alginate derivative 911 can also inhibit effectively the activity of HIV-1 reverse transcriptase, in addition to its inhibition of virus adsorption [61]. 
In conclusion, marine polysaccharides, especially the seaweed polysaccharides, can interfere with virus replication enzymes [61,67] or other potential targets within the host cells [37], so as to inhibit the virus transcription and replication processes.

\subsection{Improvement of Host Antiviral Immune Responses}

The virus infection can induce the antiviral immune responses of host cells, of which the type I interferon system (IFN- $\alpha / \beta$ system) is the cell's first line of defense against viral infections. Moreover, the host NK cells and macrophages also play important roles in these processes. Therefore, if marine polysaccharides can activate NK cells and macrophages, or stimulate the generation of antiviral immune factors, they will be able to indirectly inhibit virus replication and accelerate the process of viral clearance.

Turner $e$ t al. found that $\lambda$-carrageenan polysaccharides could significantly induce the synthesis of type I interferon in mice after being treated with carrageenan for eight hours [107]. Zhou et al. reported that the $\lambda$-carrageenan polysaccharides prepared by microwave degradation could significantly improve the activity of NK cells, and enhance the proliferation rate of lymphocyte [108]. Moreover, Yuan et al. noted that carrageenan oligosaccharides could also enhance the activities of macrophages and NK cells and increase the expression of IL-2 and TNF- $\alpha$ [109]. Therefore, the antiviral effects of carrageenans in vivo might also be related to their stimulating effects on host immune system.

Moreover, certain polysaccharides derived from brown algae and green algae can also improve the antiviral immune responses of host. The sulfated polymannuroguluronate (SPMG) derived from brown algae was reported to be able to significantly improve the humoral and cellular immune functions to indirectly inhibit the replication of HIV and HBV [62,66]. Xin et al. noted that the alginate polysaccharide drug 911 could enhance the proliferative response of $\mathrm{T}$ and B lymphocytes in mice, and strengthen the capacity of spleen cells to produce IL-2 and hemolysin antibodies [62]. In addition, water-soluble sulfated polysaccharides (SP) extracted from Enteromorpha prolifera were reported to be able to increase the IFN- $\gamma$ and IL-2 secretions, which suggested that SP could activate T cells by upregulating Th-1 response [110]. In a word, several marine polysaccharides can inhibit virus replication and accelerate the process of viral clearance indirectly through activating the immune system.

\section{Conclusions}

During the last decade, numerous bioactive polysaccharides with interesting functional properties have been discovered from marine organisms [111]. Marine polysaccharides, especially the sulfated polysaccharides derived from marine algae, often possess good inhibitory effects on a variety of viruses [18,112]. This review mainly focuses on the antiviral activities and mechanisms of marine polysaccharides, which is expected to attract more interest for future explorations. The antiviral activities of most marine polysaccharides are usually related to the specific sugar structure, molecular weights and their degree of sulfation. Marine polysaccharides can inhibit the replication of viruses through interfering with a few steps in virus life cycle or improving the host antiviral immune responses to accelerate the process of viral clearance. Despite having good antiviral effects, marine polysaccharides are structurally diverse and heterogeneous, which makes studies of their structures challenging, and may also have hindered their development as therapeutic agents to date [18]. 
In conclusion, marine polysaccharides, especially the polysaccharides derived from seaweed, have many advantages, such as relatively low production costs, broad spectrum of antiviral properties, low cytotoxicity, and wide acceptability, which suggest marine polysaccharides merit further investigation as promising antivirals that can be used alone or in combination with existing drugs [113]. Until now, most of the studies on antiviral effects of marine polysaccharides have been observed in vitro or in mouse model systems. Therefore, further studies are needed in order to investigate their antiviral activities in human subjects [60]. Moreover, the structure-activity relationships and the underlying molecular mechanisms of antiviral actions of marine polysaccharides need to be understood precisely and elucidated clearly by intensive studies in the future [111].

\section{Acknowledgments}

We thank Lijuan Zhang (Ocean University of China, China) for her helpful advice and critical readings of the manuscript. This work was supported in part by the Program for Changjiang Scholars and Innovative Research Team in University (IRT0944), the National Natural Science Foundation of China (31271923), Shandong Provincial Natural Science Foundation (ZR2011HQ012), the Fundamental Research Funds for the Central Universities (201113013), the Special Fund for Marine Scientific Research in the Public Interest (201005024), and Qingdao science and technology development project (12-1-4-1-(20)-jch).

\section{References}

1. De Clercq, E. Antiviral drugs in current chemical reviews. J. Clin. Virol. 2004, 30, 115-133.

2. Laurienzo, P. Marine polysaccharides in pharmaceutical applications: An overview. Mar. Drugs 2010, 8, 2435-2465.

3. Jiao, G.L.; Yu, G.L.; Wang, W.; Zhao, X.L.; Zhang, J.Z.; Stephen, H.E. Properties of polysaccharides in several seaweeds from Atlantic Canada and their potential anti-influenza viral activities. J. Ocean Univ. China 2012, 11, 205-212.

4. Huang, Y.L.; Zheng, T.L. Studies on polysaccharides from marine organism, a review. Mar. Sci. 2004, 28, 58-61.

5. Singh, K.; Ray, A.R. Biomedical applications of chitin, chitosan, and their derivatives. J. Macromol. Sci. 2000, 40, 69-83.

6. Zierer, M.S.; Mourao, P.S. A wide diversity of sulfated polysaccharides are synthesized by different species of marine sponges. Carbohydr. Res. 2000, 328, 209-216.

7. Ngo, D.N.; Kim, M.M.; Kim, S.K. Chitin oligosaccharides inhibit oxidative stress in live cells. Carbohydr. Polym. 2008, 74, 228-234.

8. Burrows, F.; Louime, C.; Abazinge, M.; Onokpise, O. Extraction and evaluation of chitin from crub exoskeleton as a seed fungicide and plant growth enhancer. Am. Eurasian J. Agric. Environ. Sci. 2007, 2, 103-111.

9. Kim, S.K.; Nghiep, N.D.; Rajapakse, N. Therapeutic prospectives of chitin, chitosan and their derivatives. J. Chitin Chitosan 2006, 11, 1-10.

10. Jayakumar, R.; New, N.; Nagagama, H.; Furuike, T.; Tamura, H. Synthesis, characterization and biospecific degradation behavior of sulfated chitin. Macromol. Symp. 2008, 264, 163-167. 
11. Suwan, J.; Zhang, Z.; Li, B.; Vongchan, P.; Meepowpan, P.; Zhang, F.; Mousa, S.A.; Mousa, S.; Premanode, B.; Kongtawelert, P.; et al. Sulfonation of papain-treated chitosan and its mechanism for anticoagulant activity. Carbohydr. Res. 2009, 344, 1190-1196.

12. He, Q.; Ao, Q.; Wang, A.; Gong, Y.; Zhao, N.; Zhang, X. In vitro cytotoxicity and protein drug release properties of chitosan/heparin microspheres. Tsinghua Sci. Technol. 2007, 12, 361-365.

13. Thierry, B.; Merhi, Y.; Silver, J.; Tabrizian, M. Biodegradable membrane-covered stent from chitosan-based polymers. J. Biomed. Mater. Res. 2005, 75, 556-566.

14. Prabaharan, M.; Reis, R.L.; Mano, J.F. Carboxymethyl chitosan-graft-phosphatidylethanolamine: Amphiphilic matrices for controlled drug delivery. React. Funct. Polym. 2007, 67, 43-52.

15. Gonzalez, R.P.; Leyva, A.; Moraes, M.O. Shark cartilage as source of antiangiogenic compounds: From basic to clinical research. Biol. Pharm. Bull. 2001, 24, 1097-1101.

16. Cimino, P.; Bifulco, G.; Casapullo, A.; Bruno, I.; Gomez-Paloma, L.; Riccio, R. Isolation and NMR characterization of rosacelose, a novel sulfated polysaccharide from the sponge Mixylla rosacea. Carbohydr. Res. 2001, 334, 39-47.

17. Huang, Y.L.; Zheng, Z.H.; Su, W.J.; Chen, F.; Wu, P.R.; Fang, J.R. Studies on the chemical composition of sea cucumber mensamar intercede III. Immunomodulative effects of PMI-1. Mar. Sci. Bull. 2001, 20, 88-91.

18. Jiao, G.; Yu, G.; Zhang, J.; Ewart, S.H. Chemical structures and bioactivities of sulphated polysaccharides from marine algae. Mar. Drugs 2011, 9, 196-223.

19. Rioux, L.E.; Turgeon, S.L.; Beaulieu, M. Characterization of polysaccharides extracted from brown seaweeds. Carbohydr. Polym. 2007, 69, 530-537.

20. Mabeau, S.; Kloareg, B. Isolation and analysis of the cell walls of brown algae: Fucus spiralis, F. ceranoides, F. vesiculosus, F. serratus, Bifurcaria bifurcata and Laminaria digitata. J. Exp. Bot. 1986, 38, 1573-1580.

21. Lu, F.; Wei, X.; Zhao, R. Fucoidans: Structure and bioactivity. Molecules 2008, 13, 1671-1695.

22. Knutsen, S.H.; Myslabodski, D.E.; Larsen, B.; Usov, A.I. A modified system of nomenclature for red algal galactans. Bot. Mar. 1994, 37, 163-170.

23. Liu, X.; Xu, F.; Shao, C.; She, Z.; Lin, Y.; Chan, W.L. Bioactive Metabolites from Marine Microorganisms. In Studies in Natural Products Chemistry; Atta-ur-Rahman, F.R.S., Ed.; Elsevier Science Publishers: Amsterdam, The Netherlands, 2008; pp. 197-310.

24. Debbab, A.; Aly, A.H.; Lin, W.H.; Proksch, P. Bioactive compounds from marine bacteria and fungi. Microb. Biotechnol. 2010, 3, 544-563.

25. Waters, A.L.; Hill, R.T.; Place, A.R.; Hamann, M.T. The expanding role of marine microbes in pharmaceutical development. Curr. Opin. Biotechnol. 2010, 21, 780-786.

26. Thomas, T.R.A.; Kaulekar, D.P.; Lokabarathi, P.A. Marine drugs from sponge microbe association: A review. Mar. Drugs 2010, 8, 1417-1468.

27. Zheng, T.L.; Hong, H.S.; Wang, F.; Maskaoui, K.; Su, J.; Tian, Y. The distribution characters of bacteria $\beta$-glucosidase activity in the Taiwan Strait. Mar. Pollut. Bull. 2002, 45, 168-176.

28. Lee, J.B.; Hayashi, T.; Hayashi, K.; Sankawa, U. Structural analysis of calcium spirulan (Ca-SP)-derived oligosaccharides using electrospray ionization mass spectrometry. J. Nat. Prod. 2000, 63, 136-138. 
29. Roger, O.; Kervarec, N.; Ratiskol, J.; Colliec-Jouault, S.; Chevolot, L. Structural studies of the main exopolysaccharide produced by the deep-sea bacterium Alteromonas infernus. Carbohydr. Res. 2004, 339, 2371-2380.

30. McCandless, E.L.; Craigie, J.S. Sulphated polysaccharides in red and brown algae. Planta 1979, 112, 201-212.

31. Lahaye, M. Developments on gelling algal galactans, their structure and physico-chemistry. J. Appl. Phycol. 2001, 13, 173-184.

32. Chiu, Y.H.; Chan, Y.L.; Tsai, L.W.; Li, T.L.; Wu, C.J. Prevention of human enterovirus 71 infection by kappa carrageenan. Antivir. Res. 2012, 95, 128-134.

33. Talarico, L.B.; Damonte, E.B. Interference in dengue virus adsorption and uncoating by carrageenans. Virology 2007, 363, 473-485.

34. Grassauer, A.; Weinmuellner, R.; Meier, C.; Pretsch, A.; Prieschl-Grassauer, E.; Unger, H. Iota-Carrageenan is a potent inhibitor of rhinovirus infection. Virol. J. 2008, 5, 107.

35. Carlucci, M.J.; Scolaro, L.A.; Noseda, M.D.; Cerezo, A.S.; Damonte, E.B. Protective effect of a natural carrageenan on genital herpes simplex virus infection in mice. Antivir. Res. 2004, 64, 137-141.

36. Leibbrandt, A.; Meier, C.; König-Schuster, M.; Weinmüllner, R.; Kalthoff, D.; Pflugfelder, B.; Graf, P.; Frank-Gehrke, B.; Beer, M.; Fazekas, T.; et al. Iota-carrageenan is a potent inhibitor of influenza A virus infection. PLoS One 2010, 5, e14320.

37. Talarico, L.B.; Noseda, M.D.; Ducatti, D.R.B.; Duarte, M.E.; Damonte, E.B. Differential inhibition of dengue virus infection in mammalian and mosquito cells by iota-carrageenan. J. Gen. Virol. 2011, 92, 1332-1342.

38. Buck, C.B.; Thompson, C.D.; Roberts, J.N.; Muller, M.; Lowy, D.R.; Schiller, J.T. Carrageenan is a potent inhibitor of papillomavirus infection. PLoS Pathog. 2006, 2, e69.

39. Yamada, T.; Ogamo, A.; Saito, T.; Uchiyama, H.; Nakagawa, Y. Preparation of $O$-acylated low-molecular-weight carrageenans with potent anti-HIV activity and low anticoagulant effect. Carbohydr. Polym. 2000, 41, 115-120.

40. Yamada, T.; Ogamo, A.; Saito, T.; Watanabe, J.; Uchiyama, H.; Nakagawa, Y. Preparation and anti-HIV activity of low-molecular-weight carrageenans and their sulfated derivatives. Carbohydr. Polym. 1997, 32, 51-55.

41. Ji, J.; Wang, L.C.; Wu, H.; Luan, H.M. Bio-function summary of marine oligosaccharides. Int. J. Biol. Sci. 2011, 3, 74-86.

42. Tang, F.; Chen, F.; Li, F. Preparation and potential in vivo anti-influenza virus activity of low molecular-weight $\kappa$-carrageenans and their derivatives. J. Appl. Polym. Sci. 2012, doi:10.1002/app.37502.

43. Wang, W.; Zhang, P.; Hao, C.; Zhang, X.E.; Cui, Z.Q.; Guan, H.S. In vitro inhibitory effect of carrageenan oligosaccharide on influenza A H1N1 virus. Antivir. Res. 2011, 92, 237-246.

44. Wang, W.; Zhang, P.; Yu, G.L.; Li, C.X.; Hao, C.; Qi, X.; Zhang, L.J.; Guan, H.S. Preparation and anti-influenza $\mathrm{A}$ virus activity of $\kappa$-carrageenan oligosaccharide and its sulphated derivatives. Food Chem. 2012, 133, 880-888.

45. Damonte, E.B.; Matulewicz, M.C.; Cerezo, A.S. Sulfated seaweed polysaccharides as antiviral agents. Curr. Med. Chem. 2004, 11, 2399-2419. 
46. Carlucci, M.J.; Ciancia, M.; Matulewicz, M.C.; Cerezo, A.S.; Damonte, E.B. Antiherpetic activity and mode of action of natural carrageenans of diverse structural types. Antivir. Res. 1999, 43, 93-102.

47. Girond, S.; Crance, J.M.; van Cuyck-Gandre, H.; Renaudet, J.; Deloince, R. Antiviral activity of carrageenan on hepatitis A virus replication in cell culture. Res. Virol. 1991, 142, 261-270.

48. Talarico, L.B.; Pujol, C.A.; Zibetti, R.G.; Faría, P.C.; Noseda, M.D.; Duarte, M.E.; Damonte, E.B. The antiviral activity of sulfated polysaccharides against dengue virus is dependent on virus serotype and host cell. Antivir. Res. 2005, 66, 103-110.

49. Nelson, R.M.; Cecconi, O.; Roberts, W.G.; Aruffo, A.; Linhardt, R.J.; Bevilacqua, M.P. Heparin oligosaccharides bind L- and P-selectin and inhibit acute inflammation. Blood 1993, 82, 3253-3258.

50. Douglas, M.S.; Ali, S.; Rix, D.A.; Zhang, J.G.; Kirby, J.A. Endothelial production of MCP-1: Modulation by heparin and consequences for mononuclear cell activation. Immunology 1997, 92, $512-518$.

51. Yu, L.; Garg, H.G.; Li, B.; Linhardt, R.J.; Hales, C.A. Antitumor effect of butanoylated heparin with low anticoagulant activity on lung cancer growth in mice and rats. Curr. Cancer Drug Targets 2010, 10, 229-241.

52. Copeland, R.; Balasubramaniam, A.; Tiwari, V.; Zhang, F.; Bridges, A.; Linhardt, R.J.; Shukla, D.; Liu, J. Using a 3-O-sulfated heparin octasaccharide to inhibit the entry of herpes simplex virus type 1. Biochemistry 2008, 47, 5774-5783.

53. Ramos-Kuri, M.; Barron Romero, B.L.; Aguilar-Setien, A. Inhibition of three alpha herpes viruses (herpes simplex 1 and 2 and pseudo rabies virus) by heparin, heparan and other sulfated polyelectrolytes. Arch. Med. Res. 1996, 27, 43-48.

54. Neyts, J.; Snoeck, R.; Schols, D.; Balzarini, J.; Esko, J.D.; van Schepdael, A.; de Clercq, E. Sulfated polymers inhibit the interaction of human cytomegalovirus with cell surface heparan sulfate. Virology 1992, 189, 48-58.

55. Okazaki, K.; Matsuzaki, T.; Sugahara, Y.; Okada, J.; Hasebe, M.; Iwamura, Y.; Ohnishi, M.; Kanno, T.; Shimizu, M.; Honda, E. BHV-1 adsorption is mediated by the interaction of glycoprotein gIII with heparinlike moiety on the cell surface. Virology 1991, 181, 666-670.

56. Kari, B.; Gehrz, R. A human cytomegalovirus glycoprotein complex designated gC-II is a major heparin-binding component of the envelope. J. Virol. 1992, 66, 1761-1764.

57. Madeleine, L.M.; Reinhard, G. Dextran sulfate inhibits the fusion of influenza virus with model membranes, and suppresses influenza virus replication in vivo. Antivir. Res. 1990, 14, 39-50.

58. Witvrouw, M.; Schols, D.; Andrei, G.; Snoeck, R.; Hosoya, M.; Pauwels, R.; Balzarini, J.; de Clercq, E. Antiviral activity of low-MW dextran sulphate (derived from dextran MW 1000) compared to dextran sulphate samples of higher MW. Antivir. Chem. Chemother. 1991, 2, 171-179.

59. Ivanova, V.; Rouseva, R.; Kolarova, M.; Serkedjieva, J.; Rachev, R.; Manolova, N. Isolation of a polysaccharide with antiviral effect from Ulva lactuca. Prep. Biochem. 1994, 24, 83-97.

60. Vo, T.-S.; Kim, S.-K. Potential anti-HIV agents from marine resources: An overview. Mar. Drugs 2010, 8, 2871-2892. 
61. Xin, X.L.; Geng, M.Y.; Guan, H.S.; Li, Z.L. Study on the mechanism of inhibitory action of 911 on replication of HIV-1 in vitro. Chin. J. Mar. Drugs 2000, 19, 15-18.

62. Xin, X.L.; Ding, H.; Geng, M.Y.; Liang, P.F.; Li, Y.X.; Guan, H.S. Studies of the anti-AIDS effects of marine polysaccharide drug 911 and its related mechanisms of action. Chin. J. Mar. Drugs 2000, 6, 4-8.

63. Geng, M.Y.; Li, F.C.; Xin, X.L.; Li, J.; Yan, Z.W.; Guan, H.S. The potential molecular targets of marine sulfated polymannuroguluronate interfering with HIV-1 entry Interaction between SPMG and HIV-1 gp120 and CD4 molecule. Antivir. Res. 2003, 59, 127-135.

64. Miao, B.C.; Geng, M.Y.; Li, J.; Li, F.; Chen, H.; Guan, H.S.; Ding, J. Sulfated polymannuroguluronate, a novel anti-acquired immune deficiency syndrome (AIDS) drug candidate, targeting CD4 in lymphocytes. Biochem. Pharmacol. 2004, 68, 641-649.

65. Liu, H.Y.; Geng, M.Y.; Xin, X.L.; Li, F.C.; Chen, H.X.; Guan, H.S.; Ding, J. Multiple and multivalent interactions of novel anti-AIDS drug candidates, sulfated polymannuronate (SPMG)-derived oligosaccharides, with gp120 and their anti-HIV activities. Glycobiology 2005, $15,501-510$.

66. Jiang, B.F.; Xu, X.F.; Li, L.; Yuan, W. Study on "911" anti-HBV effect in HepG2.2.15 cells culture. Mod. Prev. Med. 2003, 30, 517-518.

67. Queiroz, K.C.S.; Medeiros, V.P.; Queiroz, L.S.; Abreu, L.R.D.; Rocha, H.A.O.; Ferreira, C.V.; Juca, M.B.; Aoyama, H.; Leite, E.L. Inhibition of reverse transcriptase activity of HIV by polysaccharides of brown algae. Biomed. Pharmacother. 2008, 62, 303-307.

68. Hidari, K.I.P.J.; Takahashi, N.; Arihara, M.; Nagaoka, M.; Morita, K.; Suzuki, T. Structure and anti-dengue virus activity of sulfated polysaccharide from a marine alga. Biochem. Biophys. Res. Commun. 2008, 376, 91-95.

69. Akamatsu, E.; Shimanaga, M.; Kamei, Y. Isolation of an anti-influenza virus substance, MC26 from a marine brown alga Sargassum piluliferum and its antiviral activity against influenza virus. Coast. Bioenviron. 2003, 1, 29-34.

70. Muto, S.; Niimura, K.; Oohara, M.; Oguchi, Y.; Matsunaga, K.; Hirose, K.; Kakuchi, J.; Sugita, N.; Furusho, T. Polysaccharides from marine algae and antiviral drugs containing the same as active ingredients. Eur. Patent EP295956, 21 December 1988.

71. Yu, N.; Liu, S.; Han, J.J.; Sun, F.S. The depressive effect of glycosaminoglycan from scallop on type-I herpes simplex virus. Acta Acad. Med. Qingdao Univ. 2008, 2, 111-114.

72. Zhang, H.F.; Li, J.B.; Hou, G.; Huang, D.N. Study on the inhibition effects of Perna viridis polysaccharides on influenza virus reproduction in MDCK cell cultures. Mod. Med. J. China 2008, 5, 4-7.

73. Wu, S.M.; Xu, W.M.; Xu, Z.; Lin, F.; Sheng, L. Antiviral effect of oyster extract compound capsule in ducks infected by DHBV. Chin. Pharm. J. 1996, 31, 304-307.

74. Woo, E.R.; Kim, W.S.; Kim, Y.S. Virus-cell fusion inhibitory activity for the polysaccharides from various Korean edible clams. Arch. Pharm. Res. 2001, 24, 514-517.

75. Davis, R.; Zivanovic, S.; D'Souza, D.H.; Davidson, M.P. Effectiveness of chitosan on the inactivation of enteric viral surrogates. Food Microbiol. 2012, 32, 57-62.

76. Pospieszny, H.; Chirkov, S.; Atabekov, J. Induction of antiviral resistance in plants by chitosan. Plant Sci. 1991, 79, 63-68. 
77. Davydova, V.N.; Nagorskaia, V.P.; Gorbach, V.I.; Kalitnik, A.A.; Reunov, A.V.; Solov'eva, T.F.; Ermak, I.M. Chitosan antiviral activity: Dependence on structure and depolymerization method. Appl. Biochem. Microbiol. 2011, 47, 113-118.

78. Chirkov, S.N. The antiviral activity of chitosan (review). Appl. Biochem. Microbiol. 2002, 38, 1-8.

79. Kulikov, S.N.; Chirkov, S.N.; Il'ina, A.V.; Lopatin, S.A.; Varlamov, V.P. Effect of the molecular weight of chitosan on its antiviral activity in plants. Prik. Biokhim. Mikrobiol. 2006, 42, 224-228.

80. Sosa, M.A.; Fazely, F.; Koch, J.A.; Vercellotti, S.V.; Ruprecht, R.M. N-carboxymethyl chitosan- $N, O$-sulfate as an anti-HIV-1 agent. Biochem. Biophys. Res. Commun. 1991, 174, 489-496.

81. Nishimura, S.I.; Kai, H.; Shinada, K.; Yoshida, T.; Tokura, S.; Kurita, K.; Nakashima, H.; Yamamoto, N.; Uryu, T. Regioselective syntheses of sulfated polysaccharides: Specific anti-HIV-1 activity of novel chitin sulfates. Carbohydr. Res. 1998, 306, 427-433.

82. Dou, J.L.; Tan, C.Y.; Du, Y.G.; Bai, X.F.; Wang, K.Y.; Ma, X.J. Effects of chitooligosaccharides on rabbit neutrophils in vitro. Carbohydr. Polym. 2007, 69, 209-213.

83. Jeon, Y.J.; Kim, S.K. Continuous production of chitooligosaccharides using a dual reactor system. Process Biochem. 2000, 35, 623-632.

84. Jeon, Y.J.; Kim, S.K. Production of chitooligosaccharides using ultrafiltration membrane reactor and their antibacterial activity. Carbohydr. Polym. 2000, 41, 133-141.

85. Artan, M.; Karadeniz, F.; Karagozlu, M.Z.; Kim, M.M.; Kim, S.K. Anti-HIV-1 activity of low molecular weight sulfated chitooligosaccharides. Carbohydr. Res. 2010, 345, 656-662.

86. Pospieszny, H.; Atabekov, J.G. Effect of chitosan on the hypersensitive reaction of bean to alfalfa mosaic virus. Plant Sci. 1989, 62, 29-31.

87. Guan, H.S. New drug_Polymannuronic acid propyl sulfates. China Patent 93100608.2, 6 July 1994.

88. Guan, H.S. New drug-polymeric mannuronic acids sulfate. China Patent 95110396.2, 2 October 1996.

89. Wang, W.; Yu, G.L.; Hao, C.; Guan, H.S. The application of one kind of oligomeric mannuronic acid in the preparation of anti-influenza A H1N1 virus drugs. China Patent 201110408962.7, 9 December 2011.

90. Wang, W.; Li, C.X.; Guan, H.S.; Yu, G.L.; Wang, S.X. The application of polymannuronic acid propyl sulfate in the preparation of drugs against influenza A (H1N1) virus. China Patent 201210201876.3, 18 June 2012.

91. Kanekiyo, K.; Hayashi, K.; Takenaka, H.; Lee, J.B.; Hayashi, T. Anti-herpes simplex virus target of an acidic polysaccharide, nostoflan, from the edible blue-green alga Nostoc flagelliforme. Biol. Pharm. Bull. 2007, 30, 1573-1575.

92. Kim, M.; Yim, J.H.; Kim, S.Y.; Kim, H.S.; Lee, W.G.; Kim, S.J.; Kang, P.S.; Lee, C.K. In vitro inhibition of influenza A virus infection by marine microalga-derived sulfated polysaccharide p-KG03. Antivir. Res. 2012, 93, 253-259.

93. Carlucci, M.J.; Scolaro, L.A.; Damonte, E.B. Herpes simplex virus type 1variants arising after selection with an antiviral carageenan: Lack of correlation between drug susceptibility and syn phenotype. J. Med. Virol. 2002, 68, 92-98. 
94. Harden, E.A.; Falshaw, R.; Carnachan, S.M.; Kern, E.R.; Prichard, M.N. Virucidal activity of polysaccharide extracts from four algal species against herpes simplex virus. Antivir. Res. 2009, 83, 282-289.

95. Su, X.W.; Zivanovic, S.; D’Souza, D.H. Effect of chitosan on the infectivity of murine norovirus, feline calicivirus, and bacteriophage MS2. J. Food Protect. 2009, 72, 2623-2628.

96. Mazumder, S.; Ghosal, P.K.; Pujol, C.A.; Carlucci, M.J.; Damonte, E.B.; Ray, B. Isolation, chemical investigation and antiviral activity of polysaccharides from Gracilaria corticata (Gracilariaceae, Rhodophyta). Int. J. Biol. Macromol. 2002, 31, 87-95.

97. Carlucci, M.J.; Pujol, C.A.; Ciancia, M.; Noseda, M.D.; Matulewicz, M.C.; Damonte, E.B.; Cerezo, A.S. Antiherpetic and anticoagulant properties of carrageenans from the red seaweed Gigartina skottsbergii and their cyclized derivatives: Correlation between structure and biological activity. Int. J. Biol. Macromol. 1997, 20, 97-105.

98. Carlucci, M.J.; Scolaro, L.A.; Damonte, E.B. Inhibitory action of natural carrageenans on herpes simplex virus infection of mouse astrocytes. Chemotheraphy 1999, 45, 429-436.

99. Trinchero, J.; Ponce, N.M.; Córdoba, O.L.; Flores, M.L.; Pampuro, S.; Stortz, C.A.; Salomón, H.; Turk, G. Antiretroviral activity of fucoidans extracted from the brown seaweed Adenocystis utricularis. Phytother. Res. 2009, 23, 707-712.

100. Feldman, S.C.; Reynaldi, S.; Stortz, C.A.; Cerezo, A.S.; Damonte, E.B. Antiviral properties of fucoidan fractions from Leathesia difformis. Phytomedcine 1999, 6, 335-340.

101. Majczak, G.A.H.; Richartz, R.R.T.B.; Duarte, M.E.R.; Noseda, M.D. Antiherpetic Activity of Heterofucans Isolated from Sargassum stenophyllum (Fucales, Phaeophyta). In Proceedings of the 17th International Seaweed Symposium, Cape Town, South Africa, 28 January-2 February 2001; Chapman, A.R.O., Anderson, R.J., Vreeland, V.J., Davison, I.R., Eds.; Oxford University Press: Oxford, UK, 2003; pp. 169-174.

102. Ponce, N.M.A.; Pujol, C.A.; Damonte, E.B.; Flores, M.L.; Stortz, C.A. Fucoidans from the brown seaweed Adenocystis utricularis: Extraction methods, antiviral activity and structural studies. Carbohydr. Res. 2003, 338, 153-165.

103. Baba, M.; Snoeck, R.; Pauwels, R.; de Clercq, E. Sulfated polysaccharides are potent and selective inhibitors of various enveloped viruses, including herpes simplex virus, cytomegalovirus, vesicular stomatitis virus, and human immunodeficiency virus. Antimicrob. Agents Chemother. 1988, 32, 1742-1745.

104. Mercer, J.; Schelhaas, M.; Helenius, A. Virus entry by endocytosis. Annu. Rev. Biochem. 2010, 79, 803-833.

105. Talarico, L.B.; Duarte, M.E.R.; Zibetti, R.G.M.; Noseda, M.D.; Damonte, E.B. An algal-derived DL-galactan hybrid is an efficient preventing agent for in vitro dengue virus infection. Planta Med. 2007, 73, 1464-1468.

106. González, M.E.; Alarcón, B.; Carrasco, L. Polysaccharides as antiviral agents: Antiviral activity of carrageenan. Antimicrob. Agents Chemother. 1987, 31, 1388-1393.

107. Turner, E.V.; Sonnenfeld, G. Interferon induction by the immunomodulating polyanion Lambda carrageenan. Infect. Immun. 1979, 25, 467-469. 
108. Zhou, G.; Sun, Y.P.; Xin, H.; Zhang, Y.; Li, Z.; Xu, Z. In vivo antitumor and immunomodulation activities of different molecular weight lambda-carrageenans from Chondrus ocellatus. Pharmacol. Res. 2004, 50, 47-53.

109. Yuan, H.M.; Song, J.M.; Li, X.G.; Li, X.G.; Li, N.; Dai, J.C. Immunomodulation and antitumor activity of $\kappa$-carrageenan oligosaccharides. Cancer Lett. 2006, 243, 228-234.

110. Kim, J.-K.; Cho, M.L.; Karnjanapratum, S.; Shin, I.-S.; You, S.G. In vitro and in vivo immunomodulatory activity of sulfated polysaccharides from Enteromorpha prolifera. Int. J. Biol. Macromol. 2011, 49, 1051-1058.

111. Patel, S. Therapeutic importance of sulfated polysaccharides from seaweeds: Updating the recent findings. 3 Biotech 2012, 2, 171-185.

112. Witvrouw, M.; de Clercq, E. Sulfated polysaccharides extracted from sea algae as potential antiviral drugs. Gen. Pharm. 1997, 29, 497-511.

113. Ghosh, T.; Chattopadhyay, K.; Marschall, M.; Karmakar, P.; Mandal, P.; Ray, B. Focus on antivirally active sulfated polysaccharides: From structure-activity analysis to clinical evaluation. Glycobiology 2009, 19, 2-15.

Samples Availability: Available from the authors.

(C) 2012 by the authors; licensee MDPI, Basel, Switzerland. This article is an open access article distributed under the terms and conditions of the Creative Commons Attribution license (http://creativecommons.org/licenses/by/3.0/). 